\title{
Upper functions for plane Brownian windings
}

\section{EUGENE A. DOROFEEV}

St Petersburg Electrotechnical Institute, Higher Maths-2, St Petersburg, Russia. E-mail: dorofeev@pdmi.ras.ru

We present a method leading to a number of results on upper functions for such functionals as winding angles or radius vectors of planar processes.

Keywords: continuous local martingales; Markov moments; windings of planar random process

\section{Introduction}

Problems of sharp geometry of multidimensional Brownian curves and close problems for random walks are widely studied at present. Important results were obtained in the branch of studying the asymptotics of the functionals of winding angle type of the planar Brownian motion $Z_{t}, t \geq 0$ (see, for example, Messulam and Yor (1982), Lyons and McKean (1984), Pitman and Yor (1986, 1989), Bertoin and Werner (1993) and Shi (1994, 1995). Limit theorems of various types for the winding angle $\theta_{t}$ were obtained, most of them dealing with the windings of the process $Z_{t}$ around the origin or around another Brownian particle, independent of the first one.

Following the work of Bertoin and Werner and of Shi, we try to study lim inf-lim sup behaviour of the following functionals:

$$
\begin{gathered}
\Phi_{1}(t)=\int_{0}^{t} \llbracket\left(\rho_{s} \in\left[\epsilon_{1}, \epsilon_{2}\right]\right) \mathrm{d} \theta_{s}, \\
\Phi_{2}(t)=\int_{0}^{t} \llbracket\left(\rho_{s}>\epsilon\right) \mathrm{d} \theta_{s}, \\
\Phi(t)=\int_{0}^{t} \mathrm{~d} \theta_{s}=\theta_{t},
\end{gathered}
$$

where $\rho_{s}=\left\|Z_{s}\right\|, \epsilon_{1}, \epsilon_{2}>0$ and $\theta_{s}$ is a winding angle (see Pitman and Yor (1986) for a definition). \{ ( $(\cdot)$ stands for indicator of a random event.

In the section, we recall a couple of well-known facts corresponding to martingales. We also present several standard definitions. 


\section{Preliminary remarks}

Continuous local martingales, as discussed previously by Messulam and Yor (1982, p. 356), $\log \rho_{s}$ and the winding process $\theta_{s}$ of the Wiener process $Z$, can be represented in terms of two independent linear Wiener processes $\left(\beta_{C_{s}}, \gamma_{C_{s}}\right)$, as proved by Dambis $(1965$, p. 443), after the time change

$$
C_{t}=\int_{0}^{t} \rho_{s}^{-2} \mathrm{~d} s .
$$

More definitely, for all $\epsilon_{1}>\epsilon_{2}>0$ and $\epsilon>0$ one has almost surely

$$
\begin{gathered}
\Phi_{1}(t)=\int_{0}^{C_{t}} \llbracket\left(\beta_{s} \in\left[\log \epsilon_{1}, \log \epsilon_{2}\right]\right) \mathrm{d} \gamma_{s}=w\left(\tau_{1}\left(C_{t}\right)\right), \\
\Phi_{2}(t)=\int_{0}^{C_{t}} \llbracket\left(\beta_{s}>\log \epsilon\right) \mathrm{d} \gamma_{s}=w\left(\tau_{2}\left(C_{t}\right)\right), \\
\Phi(t)=\int_{0}^{C_{t}} \mathrm{~d} \gamma_{s}=w\left(C_{t}\right),
\end{gathered}
$$

where $w$ stands for some linear Brownian motion, independent of $\beta$, and

$$
\begin{gathered}
\tau_{1}(t)=\int_{0}^{t}\left\{\left(\beta_{s} \in\left[\log \epsilon_{1}, \log \epsilon_{2}\right]\right) \mathrm{d} s,\right. \\
\tau_{2}(t)=\int_{0}^{t} \llbracket\left(\beta_{s} \in[\log \epsilon, \infty)\right) \mathrm{d} s .
\end{gathered}
$$

Moreover, for all $r>0$ with probability 1 ,

$$
C_{T(r)}=\sigma_{\log r}
$$

where $\sigma_{s}=\inf \left(u \geq 0: \beta_{u}=s\right), T(r)=\min \left(s \geq 0: \rho_{s}=r\right)$ and

$$
\frac{\log T(r)}{2 \log r} \rightarrow 1, \quad r \rightarrow \infty
$$

(Messulam and Yor 1982, p. 355).

Let $I=I(t), t \geq 0$, be a positive random functional. We call $U_{i}, i=1,2$, the upper functions of the first and second types for the functional $I$ if, respectively, almost surely

$$
\begin{gathered}
\limsup _{t \rightarrow \infty} \frac{|I(t)|}{U_{1}(t)}=0, \\
\liminf _{t \rightarrow \infty} \frac{\sup _{0 \leq s \leq t}|I(s)|}{U_{2}(t)}=0 .
\end{gathered}
$$




\section{Upper functions and lim sup theorems}

Fix any $\alpha>1$.

Lemma 3.1. Let $F$ be an increasing function and $w$ a standard linear Brownian motion. Define

$$
\sigma_{r}=\min \left\{s>0: \beta_{s}=r\right\},
$$

where $\beta$ is an independent Brownian motion.

If

$$
\sum_{k} \mathbf{P}\left(\sup _{0 \leq s \leq \sigma_{\alpha^{k}}}|w(s)| \geq F\left(\alpha^{k}\right)\right)=\infty
$$

then the random events

$$
A_{k}=\left\{\sup _{0 \leq s \leq \sigma_{\alpha^{k}}}|w(s)| \geq F\left(\alpha^{k}\right)\right\}
$$

hold infinitely often almost surely.

Proof. Consider $\sigma$-algebras

$$
\mathscr{F}_{t}=\sigma\left(\sigma\{w(s), s \geq 0\} \times \sigma\left\{\beta_{s}, 0 \leq s \leq t\right\}\right) .
$$

Events $A_{k}$ are evidently $\mathscr{F}_{\sigma_{a^{k}}}$ measurable.

We show that, in the conditions of Lemma 3.1, one has almost surely

$$
\sum_{k} \mathbf{P}\left(\sup _{0 \leq s \leq \sigma_{\alpha^{k}}}|w(s)| \geq F\left(\alpha^{k}\right) \mid \mathscr{F}_{\sigma_{\alpha^{k-1}}}\right)=\infty .
$$

According to the Borel-Cantelli-Lévy Lemma (see, for example, Shiryaev (1989, p. 553)), the last equality is sufficient for statement of Lemma 3.1.

Put

$$
\iota_{k}=\min \left\{s \geq \sigma_{\alpha^{k-1}}: \beta_{s}=0\right\} .
$$

Using the obvious inequality

$$
\sup _{0 \leq s \leq \sigma_{\alpha^{k}}}|w(s)| \geq \sup _{\ell_{k} \leq s \leq \sigma_{\alpha^{k}}}|w(s)|
$$

on the event $\left\{c_{k}<\sigma_{\alpha^{k}}\right\}$ and applying the Markov property at time $\iota_{k}$, we get

$$
\mathbf{P}\left(\sup _{0 \leq s \leq \sigma_{\alpha^{k}}}|w(s)| \geq F\left(\alpha^{k}\right) \mid \mathscr{F}_{/ k}\right) \geq \imath\left(/_{k}<\sigma_{\alpha^{k}}\right) \mathbf{P}\left(\sup _{0 \leq s \leq \sigma_{\alpha^{k}}}|w(s)+x| \geq F\left(\alpha^{k}\right)\right),
$$

where $x=w\left(/_{k}\right)$. By an inequality for Gaussian measures (Ledoux and Talagrand 1991, p. $73)$, the second term in the product is bounded from below by $\mathbf{P}\left(\sup _{0 \leq s \leq \sigma_{\alpha^{k}}}|w(s)| \geq F\left(\alpha^{k}\right)\right.$ ).

Taking now a conditional expectation given $\mathscr{F}_{\sigma_{\alpha^{k-1}}}$, we get 


$$
\begin{aligned}
\mathbf{P}\left(\sup _{\ell_{k}<s \leq \sigma_{\alpha^{k}}}|w(s)| \geq F\left(\alpha^{k}\right) \mid \mathscr{F} \sigma_{\alpha^{k-1}}\right) & \geq \mathbf{P}\left(\ell_{k}<\sigma_{\alpha^{k}} \mid \mathscr{F}_{\sigma_{\alpha^{k-1}}}\right) \mathbf{P}\left(\sup _{0 \leq s \leq \sigma_{\alpha^{k}}}|w(s)| \geq F\left(\alpha^{k}\right)\right) \\
& =\frac{\alpha-1}{\alpha} \mathbf{P}\left(\sup _{0 \leq s \leq \sigma_{\alpha^{k}}}|w(s)| \geq F\left(\alpha^{k}\right)\right) .
\end{aligned}
$$

From (3.1) we conclude that the series

$$
\sum_{k} \mathbf{P}\left(\sup _{0 \leq s \leq \sigma_{\alpha^{k}}}|w(s)| \geq F\left(\alpha^{k}\right) \mid \mathscr{F} \sigma_{\alpha^{k-1}}\right)
$$

diverges almost surely.

The proof of the lemma is complete.

By analogy, one proves Lemma 3.2.

\section{Lemma 3.2.}

(a) Let $l_{0}^{t}$ be the local time at level 0 and time $t$ of the linear Brownian motion $\beta$. If

$$
\sum_{k} \mathbf{P}\left(\sup _{0 \leq s \leq l_{0} \alpha^{k}}|w(s)| \geq F\left(\alpha^{k}\right)\right)=\infty
$$

then the random events

$$
A_{k}=\left\{\sup _{0 \leq s \leq l_{0}^{\sigma} \alpha^{k}}|w(s)| \geq F\left(\alpha^{k}\right)\right\}
$$

hold infinitely often almost surely.

(b) Let

$$
\tau_{2}(t)=\int_{0}^{t}\left\{\left(\beta_{s} \in[\log \epsilon, \infty)\right) \mathrm{d} s\right.
$$

If

$$
\sum_{k} \mathbf{P}\left(\sup _{0 \leq s \leq \tau_{2}\left(\sigma_{\alpha^{k}}\right)}|w(s)| \geq F\left(\alpha^{k}\right)\right)=\infty,
$$

then the random events

$$
A_{k}=\left\{\sup _{0 \leq s \leq \tau_{2}\left(\sigma_{\alpha^{k}}\right)}|w(s)| \geq F\left(\alpha^{k}\right)\right\}
$$

hold infinitely often almost surely.

Proof. To prove Lemma 3.2(a), one replaces $w(s)$ everywhere in the proof of Lemma 3.1 
with $w\left(l_{0}^{s}\right)$ and, to prove Lemma 3.2(b) with $w\left(\tau_{2}(s)\right)$, respectively, and uses the additivity of the functionals introduced above.

Corollary 3.1. Let $F^{(i)}, \quad i=1,2,3$ be increasing functions such that $\forall \alpha>1 \lim \sup _{t}\left\{F^{(i)}(\alpha t) / F^{(i)}(t)\right\} \leq \alpha$.

Let the series

$$
\begin{aligned}
& \sum_{k} \mathbf{P}\left(\sup _{0 \leq s \leq \sigma_{a^{k}}}|w(s)| \geq F^{(1)}\left(\alpha^{k}\right)\right) \\
& \sum_{k} \mathbf{P}\left(\sup _{0 \leq s \leq l_{0}^{\sigma} \alpha^{k}}|w(s)| \geq F^{(2)}\left(\alpha^{k}\right)\right) \\
& \sum_{k} \mathbf{P}\left(\sup _{0 \leq s \leq \tau_{2}\left(\sigma_{a^{k}}\right)}|w(s)| \geq F^{(3)}\left(\alpha^{k}\right)\right)
\end{aligned}
$$

converge (or diverge respectively), for all $\alpha>1$ simultaneously.

Then one has almost surely

$$
\begin{gathered}
\limsup _{R} \frac{\sup _{0 \leq s \leq \sigma_{R}}|w(s)|}{F^{(1)}(R)} \leq 1 \quad \text { or } \quad \geq 1, \\
\limsup _{R} \frac{\sup _{0 \leq s \leq l_{0}^{\sigma} R}|w(s)|}{F^{(2)}(R)} \leq 1 \quad \text { or } \geq 1, \\
\limsup _{R} \frac{\sup _{0 \leq_{s} \tau_{2}\left(\sigma_{R}\right)}|w(s)|}{F^{(3)}(R)} \leq 1 \quad \text { or } \quad \geq 1,
\end{gathered}
$$

according as the series (3.2) converges or diverges

Corollary 3.1 follows immediately from the Borel-Cantelli Lemma, Lemmas 3.1 and 3.2 and the monotonicity argument.

Now we are able to prove the following result.

\section{Theorem 3.1.}

(a) Let $F$ be an increasing function such that $F(x) / x \rightarrow \infty, x \rightarrow \infty$.

Then one has almost surely

$$
\limsup _{R} \frac{\left|w\left(\sigma_{R}\right)\right|}{F(R)}=0 \quad \text { or } \quad \infty
$$

according as the integral $\int_{0}^{\infty} \mathrm{d} x / F(x)$ converges or diverges.

(b) One has almost surely

$$
\limsup _{R} \frac{\left|w\left(\tau_{2}\left(\sigma_{R}\right)\right)\right|}{R \log \log R}=\frac{2}{\pi} .
$$

To prove Theorem 3.1, we need the following lemma. 
Lemma 3.3.

$$
\mathbf{P}\left(\sup _{0 \leq s \leq \tau_{2}\left(\sigma_{\tau}\right)} w(s) \geq y\right)=\frac{2}{\pi} \mathrm{e}^{-\pi y / 2 r}[1+o(1)], \quad r, y \rightarrow \infty, r / y \rightarrow 0 .
$$

Proof. We use equation (2.2.1) from Baskakova and Borodin (1992):

$$
\mathbf{P}\left(\sup _{0 \leq s \leq t} w(s) \geq y\right)=\operatorname{erfc}\left(y /(2 t)^{1 / 2}\right)=\frac{2}{\pi^{1 / 2}} \int_{y /(2 t)^{1 / 2}}^{\infty} \mathrm{e}^{-z^{2}} \mathrm{~d} z=\left(\frac{2}{\pi t}\right)^{1 / 2} \int_{y}^{\infty} \mathrm{e}^{-z^{2} t} \mathrm{~d} z .
$$

One can easily calculate the Laplace transform of distribution of $\tau_{2}\left(\sigma_{\tau}\right)$ : it satisfies

$$
\int_{0}^{\infty} \mathrm{e}^{-\lambda t} \mathbf{P}_{\tau_{2}\left(\sigma_{\tau}\right)} \mathrm{d} t \asymp \frac{1}{\cosh \left[r(2 \lambda)^{1 / 2}\right]}, \quad \lambda>0, r \rightarrow \infty .
$$

We also remark that its density is bounded at zero.

Hence, one has

$$
\begin{aligned}
\mathbf{P}\left(\sup _{0 \leq s \leq \tau_{2}\left(\sigma_{\tau}\right)} w(s) \geq y\right) & \asymp \int_{0}^{\infty} \operatorname{erfc}\left(y /(2 t)^{1 / 2}\right) \mathbf{P}_{\tau_{2}\left(\sigma_{\tau}\right)} \mathrm{d} t \\
& =\left(\frac{2}{\pi}\right)^{1 / 2} \int_{y}^{\infty} \mathrm{d} z \int_{0}^{\infty} \mathrm{e}^{-z^{2} / 2 t} \frac{\mathbf{P}_{\tau_{2}\left(\sigma_{\tau}\right)} \mathrm{d} t}{t^{1 / 2}} \\
& =\frac{1}{\pi} \int_{y}^{\infty} \mathrm{d} z \int_{0}^{\infty} \mathbf{P}_{\tau_{2}\left(\sigma_{\tau}\right)} \mathrm{d} t \int_{-\infty}^{\infty} \mathrm{e}^{-u^{2} t / 2-\mathrm{i} u z} \mathrm{~d} u \\
& =\frac{1}{\pi} \int_{y}^{\infty} \mathrm{d} z \int_{-\infty}^{\infty} \mathrm{e}^{-\mathrm{i} u z} \mathrm{~d} u \int_{0}^{\infty} \mathrm{e}^{-u^{2} t / 2} \mathbf{P}_{\tau_{2}\left(\sigma_{\tau}\right)} \mathrm{d} t \\
& =\frac{1}{\pi} \int_{y}^{\infty} \mathrm{d} z \int_{-\infty}^{\infty} \frac{\mathrm{e}^{-\mathrm{i} u z} \mathrm{~d} u}{\cosh (r u)} \\
& =\int_{y}^{\infty} \frac{\mathrm{d} z}{r \cosh (\pi z / 2 r)} \\
& =\frac{2}{\pi} \mathrm{e}^{-\pi y / 2 r}[1+o(1)],
\end{aligned}
$$

which is desired (we make use of an expression from Dwight (1961, equation (861.62))). We should observe here that $(u, t)$-dependent double integral in the second line is absolutely convergent for any $z>0$, and hence one can change the order of integration.

The lemma is proved.

Proof of Theorem 3.1. Theorem 3.1(a) is essentially Khintchin's result on the Cauchy process $w\left(\sigma_{r}\right)$.

To prove Theorem 3.1(b), recall that for any $r, y>0$ 


$$
\mathbf{P}\left(\sup _{0 \leq s \leq \tau_{2}\left(\sigma_{r}\right)} w(s) \geq y\right) \leq \mathbf{P}\left(\sup _{0 \leq s \leq \tau_{2}\left(\sigma_{r}\right)}|w(s)| \geq y\right) \leq 2 \mathbf{P}\left(\sup _{0 \leq s \leq \tau_{2}\left(\sigma_{r}\right)} w(s) \geq y\right) .
$$

Fix any $\alpha>1$.

One sees from Lemma 3.3 that the series

$$
\sum_{k} \mathbf{P}\left(\sup _{0 \leq s \leq \tau_{2}\left(\sigma_{\alpha^{k}}\right)}|w(s)| \geq \frac{2}{\pi} \alpha^{k} \log \log \alpha^{k}\right)
$$

diverges and the series

$$
\sum_{k} \mathbf{P}\left(\sup _{0 \leq s \leq \tau_{2}\left(\sigma_{\alpha^{k}}\right)}|w(s)| \geq \frac{2}{\pi} \alpha^{k}\left(\log \log \alpha^{k}+2 \log \log \log \alpha^{k}\right)\right)
$$

converges. Corollary 3.1 completes the proof of Theorem 3.1(b).

It is not difficult to derive from Theorem 3.1 that for increasing function $f$ one has almost surely

$$
\limsup _{r} \frac{\left|w\left(\sigma_{\log r}\right)\right|}{\log r f(r)}=0 \quad \text { or } \quad \infty,
$$

according as the integral $\int_{0}^{\infty} \mathrm{d} x /\{x \log x f(x)\}$ converges or diverges, and

$$
\limsup _{r} \frac{\left|w\left(\tau_{2}\left(\sigma_{\log r}\right)\right)\right|}{\log r \log \log \log r}=\frac{2}{\pi} \text {. }
$$

Now we can prove the following theorem for the first-type upper function of the Brownian winding process.

\section{Theorem 3.2.}

(a) Let $f$ be an increasing function.

Then one has almost surely

$$
\limsup _{t} \frac{|\Phi(t)|}{\log t f(t)}=0 \quad \text { or } \quad \infty,
$$

according as the integral $\int_{0}^{\infty} \mathrm{d} x /\{x \log x f(x)\}$ converges or diverges.

(b)

$$
\limsup _{t} \frac{\left|\Phi_{2}(t)\right|}{\log t \log \log \log t}=\frac{1}{\pi}
$$

Result (a) was given by Bertoin and Werner (1993), and (b) was independently obtained by Shi (1995).

Proof. From (2.2) we get

$$
\Phi(t)=w\left(C_{t}\right), \Phi(T(r))=w\left(\sigma_{\log r}\right), \Phi_{2}(T(r))=w\left(\tau_{2}\left(\sigma_{\log r}\right)\right) .
$$


We have already proved the desired statement for partial case when $t \rightarrow \infty$ along the set of moments $T(R)=\min \left\{t>0:\left\|Z_{t}\right\|=R\right\}, R>0$.

To complete the proof, one must use the monotonicity argument, as in Corollary 3.1. We recall here that almost surely $\log T(r) / 2 \log r \rightarrow 1, r \rightarrow \infty$.

Now we turn our attention to the functional

$$
\Phi_{1}(t)=\int_{0}^{C_{t}}\left\{\left(\beta_{s} \in\left[\log \epsilon_{1}, \log \epsilon_{2}\right]\right) \mathrm{d} \gamma_{s} .\right.
$$

We use the following lemma.

\section{Lemma 3.4.}

$$
\mathbf{P}\left(\sup _{0 \leq s \leq l_{0}^{\sigma \tau}} w(s) \geq y\right)=\mathrm{e}^{-y / r^{1 / 2}}
$$

Proof. One proves this lemma in the same way as Lemma 3.3, using the well-known identity $\mathbf{P}\left(l_{0}^{\sigma_{\tau}}>y\right)=\mathrm{e}^{-y / 2 r}$.

Now we get the following result.

Lemma 3.5. Almost surely

$$
\limsup _{r} \frac{1}{r^{1 / 2} \log \log r}\left|w\left(l_{0}^{\sigma_{\tau}}\right)\right|=1 .
$$

Proof. The proof is based on the fact that, according to Lemma 3.4, the series

$$
\sum_{k} \mathbf{P}\left(\sup _{0 \leq s \leq l_{0} \alpha^{2 k}} w(s) \geq F\left(\alpha^{2 k}\right)\right)
$$

converges, simultaneously for all $\alpha>1$, for

$$
F_{1}(x)=x^{1 / 2}(\log \log x+2 \log \log \log x)
$$

and diverges, respectively, for

$$
F_{2}(x)=x^{1 / 2} \log \log x
$$

Finally, one uses Corollary 3.1 as in proof of Theorem 3.1.

Corollary 3.2. Almost surely

$$
\limsup _{r} \frac{1}{(\log r)^{1 / 2} \log \log \log r}\left|w\left(\tau_{1}\left(C_{T(r)}\right)\right)\right|=\left\{\log \left(\frac{\epsilon_{2}}{\epsilon_{1}}\right)\right\}^{1 / 2} .
$$


Proof. It suffices to observe that, as was proved by Itô and McKean (1965, p. 229),

$$
\lim _{r \rightarrow \infty} \frac{\tau_{1}(r)}{l_{0}^{r}}=\log \left(\frac{\epsilon_{2}}{\epsilon_{1}}\right)
$$

and use Lemma 3.5, bearing in mind that $C_{T(r)}=\sigma_{\log r}$ and, if $t / t_{1} \rightarrow c>0, t_{1} \rightarrow \infty$, then for any function $f(t)$

$$
\left(\limsup \frac{|w(t)|}{f(t)}\right) /\left(\limsup _{t_{1}} \frac{\left|w\left(t_{1}\right)\right|}{f(t)}\right) \rightarrow c^{1 / 2},
$$

almost surely.

Now, by analogy with Theorem 3.2, we get from Corollary 3.2 one more result.

Theorem 3.3. Almost surely

$$
\limsup _{t} \frac{\left|\Phi_{1}(t)\right|}{(\log t)^{1 / 2} \log \log \log t}=\left(\frac{\log \left(\epsilon_{2} / \epsilon_{1}\right)}{2}\right)^{1 / 2} .
$$

Hence, we have completely classified the first-type upper functions for the winding functionals $\Phi$. Next, we prove one result corresponding to the second type.

\section{A lim inf result}

Lemma 4.1. Let $G$ be a positive increasing function.

Then, if

$$
\sum_{k} \mathbf{P}\left(\sup _{0 \leq s \leq l_{0} \alpha^{k}}|w(s)| \leq G\left(\alpha^{k}\right)\right)=\infty
$$

then the events

$$
A_{k}=\left\{\sup _{0 \leq s \leq l_{0} \alpha^{k}}|w(s)| \leq G\left(\alpha^{k}\right)\right\}
$$

hold infinitely often almost surely.

Before proving Lemma 4.1, we should first prove one useful inequality.

Lemma 4.2. Let $1 \leq j \leq k, j, k \in \mathbb{N}$.

Then

$$
\mathbf{P}\left(A_{j} \cap A_{k}\right) \leq \mathbf{P}\left(A_{j}\right)\left\{\mathbf{P}\left(A_{k}\right)+\alpha^{j-k}\right\} .
$$


Proof. One has

$$
\begin{aligned}
& \mathbf{P}\left(A_{j} \cap A_{k}\right)=\mathbf{P}\left(\sup _{0 \leq s \leq l_{0}^{\sigma} \alpha^{j}}|w(s)| \leq G\left(\alpha^{j}\right), \sup _{0 \leq s \leq l_{0}^{\sigma} \alpha^{k}}|w(s)| \leq G\left(\alpha^{k}\right)\right) \\
& =\int_{-G\left(\alpha^{j}\right)}^{G\left(\alpha^{j}\right)} \mathbf{P}\left(\sup _{l_{0}^{\sigma} \alpha^{j} \leq s \leq l_{0}^{\sigma} \alpha^{k}}|w(s)| \leq G\left(\alpha^{k}\right) \mid w\left(l_{0}^{\sigma_{\alpha^{j}}}\right)=u\right) \\
& \times \mathbf{P}\left(\sup _{0 \leq s \leq l_{0}^{\sigma_{\alpha j}}}|w(s)| \leq G\left(\alpha^{j}\right), w\left(l_{0}^{\sigma_{\alpha j}}\right)=\mathrm{d} u\right) .
\end{aligned}
$$

Using the well-known property of Gaussian measures proved by Ledoux and Talagrand (1991, p. 73) and the independence of $w$ and the process $\beta$ that determines local times $l_{0}^{\sigma_{\alpha^{k}}}$, one gets easily that almost surely

$$
\mathbf{P}\left(\sup _{l_{0}{ }_{l^{j}{ }^{j} \leq s \leq l_{0} \alpha^{k}}}|w(s)| \leq G\left(\alpha^{k}\right) \mid w\left(l_{0}^{\sigma_{\alpha j}}\right)=u\right) \leq \mathbf{P}\left(\sup _{l_{l_{0}{ }^{j}{ }^{j} \leq s \leq l_{0} \alpha^{k}}}|w(s)| \leq G\left(\alpha^{k}\right) \mid w\left(l_{0}^{\sigma_{\alpha j}}\right)=0\right) .
$$

Thus, using (4.2) and the independence of $w$ and $\beta$, we see that

$$
\begin{aligned}
\mathbf{P}\left(A_{j} \cap A_{k}\right) \leq & \mathbf{P}\left(\sup _{l_{0}^{\sigma} \alpha^{j} \leq s \leq l_{0} \alpha^{k}}|w(s)| \leq G\left(\alpha^{k}\right) \mid w\left(l_{0}^{\sigma_{\alpha} \alpha^{j}}\right)=0\right) \\
& \times \int_{-G\left(\alpha^{j}\right)}^{G\left(\alpha^{j}\right)} \mathbf{P}\left(\sup _{0 \leq s \leq l_{0}^{\sigma} \alpha^{j}}|w(s)| \leq G\left(\alpha^{j}\right), w\left(l_{0}^{\sigma_{\alpha^{j}}}\right)=\mathrm{d} u\right) \\
= & P\left(A_{j}\right) \mathbf{P}\left(\sup _{\substack{\sigma_{0} \alpha^{k}-l_{0} \alpha^{j} \\
0 \leq 0_{0}}}|w(s)| \leq G\left(\alpha^{k}\right)\right) .
\end{aligned}
$$

Observe that

$$
\begin{aligned}
& \mathbf{P}\left(\sup _{0 \leq s \leq l_{0}^{\sigma} \alpha^{k}-l_{0}^{\sigma} \alpha^{j}}|w(s)| \leq G\left(\alpha^{k}\right)\right)=\mathbf{P}\left(\sup _{0 \leq s \leq l_{0}^{\sigma} \alpha^{k}-l_{0} \alpha^{j}}|w(s)| \leq G\left(\alpha^{k}\right), \inf _{\sigma_{\alpha^{j}} \leq s \leq \sigma_{\alpha^{k}}}\left|\beta_{s}\right|=0\right) \\
& +\mathbf{P}\left(\inf _{\sigma_{\alpha^{j}} \leq s \leq \sigma_{\alpha^{k}}}\left|\beta_{s}\right|>0\right) \\
& =\mathbf{P}\left(\sup _{0 \leq s \leq l_{0}^{\sigma} \alpha^{k}-l_{0}^{\sigma} \alpha^{j}}|w(s)| \leq G\left(\alpha^{k}\right), \inf _{\sigma_{\alpha^{j}} \leq s \leq \sigma_{\alpha^{k}}}\left|\beta_{s}\right|=0\right) \\
& +\alpha^{j-k} \text {. }
\end{aligned}
$$

Define

$$
{ }_{j k}=\min \left\{\min \left\{s \in\left[\sigma_{\alpha^{j}}, \sigma_{\alpha^{k}}\right]:\left|\beta_{s}\right|=0\right\}, \sigma_{\alpha^{k}}\right\}
$$


Again from additivity properties and the Ledoux-Talagrand inequality we get

$$
\begin{aligned}
& \mathbf{P}\left(\sup _{0 \leq s \leq l_{0}^{\alpha^{k}}-l_{0}^{\sigma} \alpha^{j}}|w(s)| \leq G\left(\alpha^{k}\right), \min _{\sigma_{\alpha^{j}} \leq t \leq \sigma_{\alpha^{k}}}\left|\beta_{t}\right|=0\right) \\
& \leq \int_{-G\left(\alpha^{k}\right)}^{G\left(\alpha^{k}\right)} \mathbf{P}\left(\sup _{\ell_{j k} \leq s \leq l_{0} \alpha^{\alpha}}|w(s)| \leq G\left(\alpha^{k}\right) \mid w\left(/_{j k}\right)=u\right) \mathbf{P}_{w(/ j k)} \mathrm{d} u
\end{aligned}
$$

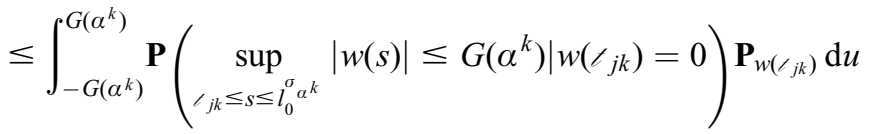

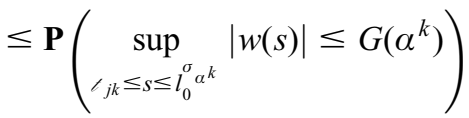

$$
\begin{aligned}
& =\mathbf{P}\left(\sup _{0 \leq s \leq l_{0}^{\sigma} \alpha^{k}}|w(s)| \leq G\left(\alpha^{k}\right)\right) \\
& =\mathbf{P}\left(A_{k}\right) .
\end{aligned}
$$

Finally, from (4.3)-(4.5) we obtain (4.1).

Now return to the proof of Lemma 4.1.

Proof of Lemma 4.1. From Lemma 4.2 we have for any $n \in \mathbb{N}$

$$
\begin{aligned}
\sum_{0 \leq j, k \leq n} \mathbf{P}\left(A_{j} \cap A_{k}\right) & \leq 2 \sum_{0 \leq j \leq k \leq n} \mathbf{P}\left(A_{j}\right)\left[\mathbf{P}\left(A_{k}\right)+\alpha^{j-k}\right] \\
& =2\left(\sum_{0 \leq j \leq n} \mathbf{P}\left(A_{j}\right)\right)^{2}+2 \sum_{0 \leq j \leq n} \mathbf{P}\left(A_{j}\right) \sum_{j \leq k \leq n} \alpha^{j-k} \\
& \leq 2\left(\sum_{0 \leq j \leq n} \mathbf{P}\left(A_{j}\right)\right)^{2}+\frac{2 \alpha}{\alpha-1} \sum_{0 \leq j \leq n} \mathbf{P}\left(A_{j}\right) \\
& \leq C\left(\sum_{0 \leq j \leq n} \mathbf{P}\left(A_{j}\right)\right)^{2},
\end{aligned}
$$

with some $C>0$, as, in conditions of Lemma $4.1, \sum_{j} \mathbf{P}\left(A_{j}\right)=\infty$. 
Applying the Kochen-Stone (1964) version of the Borel-Cantelli Lemma hence we get that

$$
\mathbf{P}\left(\sup _{0 \leq s \leq l_{0}^{\sigma} \alpha^{k}}|w(s)| \leq G\left(\alpha^{k}\right) \text { i.o. }\right) \geq C^{-1} .
$$

Finally, from the $0-1$ law we see that actually

$$
\mathbf{P}\left(\sup _{0 \leq s \leq l_{0}^{\sigma} \alpha^{k}}|w(s)| \leq G\left(\alpha^{k}\right) \text { i.o. }\right)=1
$$

thus completing the proof of Lemma 4.1. We omit a simple proof of the fact that the event in brackets belongs to the tail $\sigma$-algebra based on process $(\beta, w)$.

Now we estimate $\mathbf{P}\left(A_{k}\right)$.

\section{Lemma 4.3.}

$$
\mathbf{P}\left(\sup _{0 \leq s \leq l_{0}^{\sigma \tau}}|w(s)| \leq y\right)=\frac{y^{2}}{2 r}(1+o(1)), \quad r, y \rightarrow \infty, \frac{y}{r^{1 / 2}} \rightarrow 0 .
$$

Proof. According to equations (2.2.4) and (6.3.3) from Baskakova and Borodin (1992), we have

$$
\begin{gathered}
\mathbf{P}\left(\sup _{0 \leq s \leq t}|w(s)| \leq y\right)=1-2 \sum_{k=0}^{\infty}(-1)^{k} \operatorname{erfc}\left(\frac{y(1+2 k)}{(2 t)^{1 / 2}}\right) \\
\mathbf{P}\left(l_{0}^{\sigma_{\tau}}>y\right)=\mathrm{e}^{-y / 2 r}
\end{gathered}
$$

Thus,

$$
\begin{aligned}
\mathbf{P}\left(\sup _{0 \leq s \leq l_{0}^{\sigma \tau}}|w(s)| \leq y\right) & =\frac{1}{2 r} \int_{0}^{\infty} \mathrm{e}^{-t / 2 r} \mathrm{~d} t\left\{1-2 \sum_{k=0}^{\infty}(-1)^{k} \operatorname{erfc}\left(\frac{y(1+2 k)}{(2 t)^{1 / 2}}\right)\right\} \\
& =\frac{1}{2 r} \int_{0}^{\infty} \mathrm{e}^{-t / 2 r} \mathrm{~d} t\left(1-\frac{4}{\pi^{1 / 2}} \sum_{k=0}^{\infty}(-1)^{k} \int_{y(1+2 k) /(2 t)^{1 / 2}}^{\infty} \mathrm{e}^{-x^{2}} \mathrm{~d} x\right) \\
& =1-\frac{2}{r \pi^{1 / 2}} \sum_{k=0}^{\infty}(-1)^{k} \int_{0}^{\infty} \mathrm{e}^{-t / 2 r} \mathrm{~d} t \int_{y(1+2 k) /(2 t)^{1 / 2}}^{\infty} \mathrm{e}^{-x^{2}} \mathrm{~d} x .
\end{aligned}
$$

Changing the order of integration is correct because two integrals and the series are mutually absolutely convergent. 
Now we have

$$
\begin{aligned}
\mathbf{P}\left(\sup _{0 \leq s \leq l_{0}^{\sigma \tau}}|w(s)| \leq y\right) & =1-\frac{2}{r \pi^{1 / 2}} \sum_{k=0}^{\infty}(-1)^{k} \int_{0}^{\infty} \mathrm{e}^{-t / 2 r} \mathrm{~d} t \int_{y(1+2 k) /(2 t)^{1 / 2}}^{\infty} \mathrm{e}^{-x^{2}} \mathrm{~d} x \\
& =1-\frac{4}{\pi^{1 / 2}} \sum_{k=0}^{\infty}(-1)^{k} \int_{0}^{\infty} \mathrm{e}^{-x^{2}} \mathrm{~d} x \int_{y^{2}(1+2 k)^{2} / 4 x^{2} r}^{\infty} \mathrm{e}^{-t} \mathrm{~d} t \\
& =1-\frac{4}{\pi^{1 / 2}} \sum_{k=0}^{\infty}(-1)^{k} \int_{0}^{\infty} \mathrm{e}^{-x^{2}-y^{2}(1+2 k)^{2} / 4 x^{2} r} \mathrm{~d} x \\
& =1-2 \sum_{k=0}^{\infty}(-1)^{k} \mathrm{e}^{-y(1+2 k) / r^{1 / 2}} \\
& =1-\frac{2 \mathrm{e}^{-y / r^{1 / 2}}}{1+\mathrm{e}^{-2 y / r^{1 / 2}}} \\
& =\frac{y^{2}}{2 r}[1+o(1)], \quad r, y \rightarrow \infty, \frac{y}{r^{1 / 2}} \rightarrow 0,
\end{aligned}
$$

thus proving Lemma 4.3.

The following result is analogous to Corollary 3.1. Its proof is omitted.

Lemma 4.4. Let $G$ be an increasing function such that $\forall \alpha>1 \lim \sup _{t}\{G(\alpha t) / G(t)\} \leq \alpha$. Let the series

$$
\sum_{k} \mathbf{P}\left(\sup _{0 \leq s \leq l_{0}^{\sigma} \alpha^{k}}|w(s)| \leq G\left(\alpha^{k}\right)\right)
$$

converge (or diverge, respectively) for all $\alpha>1$ simultaneously.

Then one has almost surely



according as the series (4.6) diverges or converges.

Now, using Lemmas 4.3 and 4.4, we obtain the following corollary.

Corollary 4.3. Let $G$ be an increasing function such that $\forall \alpha>1 \lim \sup _{t}\{G(\alpha t) / G(t)\} \leq \alpha$ and $G^{2}(t)=o(t), t \rightarrow \infty$. 
Then one has almost surely

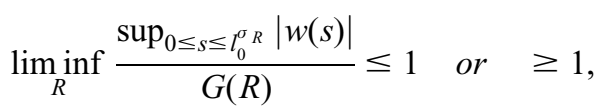

according as the integral $\int_{.}^{\infty}\left\{G^{2}(y) \mathrm{d} y\right\} / y^{2}$ diverges (or converges, respectively).

For the proof, one should only check that, for any positive constant $c>0$, the convergence of the integral is equivalent to convergence of the series (4.6):

$$
\sum_{k} \mathbf{P}\left(\sup _{0 \leq s \leq l_{0}^{\sigma} \alpha^{k}}|w(s)| \leq c G\left(\alpha^{k}\right)\right)=\sum_{k} \frac{G^{2}\left(\alpha^{k}\right)}{\alpha^{k}}\left[2 c^{2}+o(1)\right] .
$$

The estimation of probability follows from Lemma 4.3. We omit the calculations.

Finally, by analogy to Theorems 3.1 and 3.2, one obtains the following theorem.

Theorem 4.1. Let $g$ be a decreasing function such that

$$
\forall \alpha>1 \quad \lim _{t} \frac{g\left(\mathrm{e}^{\alpha t}\right)}{g\left(\mathrm{e}^{t}\right)}=1 .
$$

Then one has almost surely

$$
\liminf _{t} \frac{\sup _{0 \leq s \leq t}\left|\Phi_{1}(s)\right|}{(\log t)^{1 / 2} g^{(t)}}=0 \quad \text { or } \quad \infty,
$$

according as the integral $\int_{.}^{\infty}\left\{g^{2}(y) \mathrm{d} y\right\} /(y \log y)$ diverges or converges.

Shi (1995) found the corresponding results for both $\Phi$ and $\Phi_{2}$. The following theorem holds.

Theorem 4.2. One has almost surely

$$
\liminf _{t} \frac{\log \log \log t}{\log t} \sup _{0 \leq s \leq t}|\Phi(s)|=\frac{\pi}{4}
$$

and for any increasing function $f$ such that $\log [t / f(t)]$ is also increasing

$$
\liminf _{t} \frac{f(t)}{\log t} \sup _{0 \leq s \leq t}\left|\Phi_{2}(s)\right|=0 \quad \text { or } \quad \infty,
$$

according as the integral $\int^{\infty} \mathrm{d} y /\{y \log y f(y)\}$ diverges or converges.

\section{References}

Baskakova, P.E. and Borodin, A.N., (1992) Tables of distributions of the functionals of Brownian motion. Zapiski Nauchn. Semin. POMI RAN, 194, 8-20 (in Russian).

Bertoin, J. and Werner, W. (1993) Comportement asymptotique du nombre de tours effectués par la 
trajectoire brownienne plane. Le Seminaire de Probabilités XXVIII. Lecture Notes in Math. Berlin: Springer-Verlag.

Dambis, K.E. (1965) On decomposition of continuous submartingales. Teor. Verojatn. Prim., 10, 438448 (in Russian).

Dwight, H.B. (1961) Tables of Integrals and Other Mathematical Data. (London: Macmillan)

Itô, R. and McKean, H.P.J. (1965) Diffusion Processes and Their Sample Paths. Berlin: SpringerVerlag.

Kochen, S. and Stone, C. (1964) A note on Borel-Cantelli lemma. Illinois J. Math., 8, 248-251.

Ledoux, M. and Talagrand, M. (1991) Probability in Banach Spaces: Isoperimetry and Processes. Berlin: Springer-Verlag.

Lyons, T. and McKean, H.P. (1984) Windings of the plane Brownian motion. Adv. Math., 51, 212225.

Messulam, P. and Yor, M. (1982) On D. Williams' 'pinching method' and some applications. J. London Math. Soc., 2, 348-364.

Pitman, J. and Yor, M. (1986) Asymptotic laws of planar Brownian motion. Ann. Probab., 14, 733779 .

Pitman, J. and Yor, M. (1989) Further asymptotic laws of planar Brownian motion. Ann. Probab., 17, 965-1011.

Shi, Z. (1994) Liminf behaviors of the windings and Lévy's stochastic areas of planar Brownian motion. Le Seminaire de Probabilitiés XXVIII, pp. 122-137. Lecture Notes in Math. 1583. Berlin: Springer-Verlag.

Shi, Z. (1995) Windings of Brownian motion and random walk.

Shiryaev, A.N. (1989) Probabiliy. Moscow: Nauka.

Received September 1995 and revised February 1997 